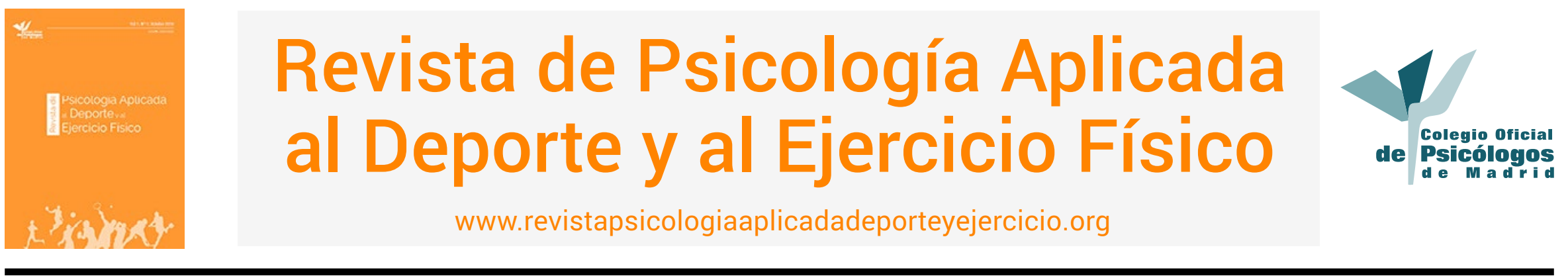

\title{
El Mindfulness como Intervención en Psicología del Deporte - Revisión sistemática
}

\author{
Víctor Contreras Pérez ${ }^{\mathrm{a}}$ Roberto Crobub \\ ${ }^{a}$ Colegio Oficial de Psicólogos de la Región de Murcia, España. ${ }^{b}$ Universidad de Murcia, España
}

RESUMEN: El objetivo del presente trabajo es realizar una revisión de estudios sobre la aplicación de mindfulness o programas de atención plena en deportistas. Para ello, se utilizaron las bases de datos de EBSCO, Dialnet, WOS y Google académico, de las cuales se seleccionó 14 artículos representativos sobre la temática comprendidos entre los años 2000 y 2018. Los resultados de las investigaciones analizadas muestran que a pesar de que existen distintos tipos de intervención con mindfulness en deportistas, los programas Mindfulness-Acceptance (MAC) y Mindfulness Sport Performance Enhancement (MSPE) son los que ofrecen una validación empírica más extendida. Dentro de los beneficios del empleo del mindfulness, destacar la reducción de la ansiedad, la mejora de competencias cognitivas atencionales y de vigilia, mayor compromiso y orientación a objetivos, con impacto todavía poco claro sobre el rendimiento deportivo, aunque genera actitudes positivas y mejoras en la calidad de vida de los deportistas. Se concluye que el mindfulness puede ser una estrategia de intervención a considerar para los psicólogos en el entrenamiento de la atención hacia los eventos mentales que surgen en el momento presente, facilitando la mejora de ciertas competencias atencionales y cognitivas, y el bienestar de los deportistas, a la vez que genera actitudes adaptativas en contextos deportivos.

PALABRAS CLAVES: Minfulness, Intervención, Deporte, Rendimiento.

\section{Mindfulness as Sport Psychology Intervention. A Systematic Review}

ABSTRACT: The purpose of this paper is to review the available studies on use of mindfulness in athletes. For this, the EBSCO, Dialnet, WOS, and Google academic databases were used, and 14 representative articles on the topic published between 2000 and 2018 were selected. The results of the reports analyzed show that while there are different types of mindfulness interventions in athletes, the Mindfulness-Acceptance (MAC) and Mindfulness Sport Performance Enhancement (MSPE) programs offer the most comprehensive empirical validation. Among the benefits of use of mindfulness, special mention should be made of reduction of anxiety, improvement in attentional cognitive skills and awareness, and increased commitment and goal orientation. Impact on sports performance is still unclear, although it generates positive attitudes and improvements in the quality of life of athletes. It is concluded that mindfulness may be an intervention strategy to be considered by psychologists for training attention to the currently arising mental events, promoting improvement of certain attentional and cognitive competences and athlete well-being, while also generating adaptive attitudes in sport contexts.

KEYWORDS: Mindfulness, Intervention, Sport, Performance.

\section{O Mindfulness como intervenção na Psicologia do desporto - Revisão sistemática}

RESUMO: O objetivo deste trabalho é realizar uma revisão de estudos sobre a aplicação de mindfulness ou programas de mindfulness em atletas. Para tal, foram utilizadas as bases de dados da EBSCO, Dialnet, WOS e Google académico, a partir das quais foram selecionados 14 artigos representativos sobre o tema, compreendidos entre os anos de 2000 e 2018. Os resultados das investigações

\footnotetext{
Víctor Contreras Pérez. Es Psicólogo y miembro de la Sección de Psicología del Deporte del Colegio Oficial de Psicólogos de la Región de Murcia Roberto Crobu. Es Psicólogo y Docente del Programa de Mindfulness MBET de la Universidad de Murcia.

Para la correspondencia de este artículo enviar a Roberto Crobu. c/ Castillo de Aledo 74 bajo b Altorreal (30506). Molina de Segura. Murcia.

E-mail: roberto@optimacoaching.es
} 
analisadas mostram que, apesar de existirem diferentes tipos de intervenção com mindfulness em atletas, os programas de MindfulnessAcceptance (MAC) e Mindfulness Sport Performance Enhancement (MSPE) são os que apresentam mais argumentos empíricos. Entre os benefícios da aplicação do mindfullness, com destaque para a redução da ansiedade, a melhoria das competências cognitivas de atenção e de vigília conduz a um maior empenho e orientação face aos objetivos, com um impacto ainda pouco claro sobre o rendimento desportivo, apesar de gerar atitudes e melhorias positivas na qualidade de vida dos atletas. Conclui-se que o mindfullness pode ser uma estratégia de intervenção a ser considerada pelos psicólogos na formação de atenção para os eventos mentais que surgem no momento presente, facilitando o aprimoramento de determinadas competências cognitivas e de atenção, bem como o bem-estar dos atletas. Ao mesmo tempo, gera atitudes adaptativas em contextos desportivos.

PALAVRAS-CHAVE: mindfullness, intervenção, desporto, desempenho.

Artículo recibido: 10/06/2018 | Artículo aceptado: 01/11/2018

En el marco de las nuevas aplicaciones en Psicología del Deporte, el uso de mindfulness resulta ser en los últimos años una herramienta de intervención novedosa enfocada a una mejora de rendimiento en los atletas (Carraça, Serpa, Palmi y Rosado, 2018).

El concepto de "mindfulness" también conocido como "atención plena", surge como la traducción al inglés de la palabra "Sati" del idioma Pali usado en el budismo (Panikkar, 1997 visto en Cepeda y Romero, 2014), el cual fue descrito en un primer momento por Kabat-Zinn (1990), que junto a otros autores lo definen como "una conciencia centrada en el presente, no elaborativa y no enjuiciadora, en la cual cada pensamiento, sentimiento o sensación que surge en el campo atencional es reconocido y aceptado tal como es" (Segal, Williams y Teasdale, 2002; Shapiro y Schartz, 1999).

Otros autores como Bishop et al. (2006) separan dos componentes en el concepto de mindfulness: por un lado, el objetivo es redireccionar, reconocer y mantener la atención hacia los eventos mentales que surgen en el momento presente. Por otro lado, el sujeto adopta una actitud de aceptación, curiosidad y apertura en el presente.

A su vez, Germer en 2005 (Cepeda y Romero, 2014), plantea que existen diferentes perspectivas de comprender el concepto de mindfulness: primero, considera que es un ejercicio de meditación; segundo, se mantiene la conciencia centrada en el presente; tercero, implica orientar la atención, a "darnos cuenta" de los acontecimientos de la experiencia presente.

Según Palmi y Solé (2016) para que un atleta consiga llegar al estado de conciencia citado puede utilizar distintas metodologías dentro de su entrenamiento, como la meditación, el yoga, mantener la consciencia en las actividades de la vida diaria, ejercicios respiratorios y de consciencia corporal entre otros.

Las técnicas de intervención con mindfulness que utiliza la Psicología se enmarcan dentro de la llamada "ola de terapias" o "terapias de tercera generación" (Hayes y Strosahl,
2004; Pérez-Álvarez, 2012), las cuales han tenido un gran desarrollo desde la década de los 90. Algunas de estas técnicas o terapias son la terapia de aceptación y compromiso (ACT) (Hayes, Strosahl y Wilson, 1999), la psicoterapia funcional analítica (FAP) (Kohlenberg y Tsai, 1991), la terapia de conducta dialéctica (DBT) (Linehan, 1993), la activación conductual (BA) (Jacobson, Martell y Dimidjian, 2001), la terapia conductual integral de pareja (IBCT) (Jacobson, Christensen, Prince, Cordova y Eldridge, 2000) y Mindfulness Based Cognitiva Therapy (MMBCT) (Segal et al., 2002).

Mindfulness pude emplearse a modo complementario a la intervención psicológica tradicional en deportistas, en este caso, implica por parte del atleta la aceptación radical de la experiencia, abandonando el control de los eventos internos y adoptando una postura contemplativa, así, este nuevo modelo de intervención puede contribuir a que los deportistas aumenten su rendimiento (Del Águila, Mañas, Franco, Gil y Montoya, 2009). Los modelos basados en la aceptación determinan que para alcanzar el rendimiento óptimo no es necesario la reducción o el control volitivo de los estados internos, sino que se requiere una aceptación del estado interno, una atención de los estímulos externos relevantes para la tarea y una entrega incondicional del esfuerzo por parte del atleta (Gardner y Moore, 2007). Según Segal et al; (2002) la práctica continuada de mindfulness disminuye la reactividad emocional (menor rumiación de los pensamientos) y por otro lado favorece la apertura a la experiencia (menor evitación de la experiencia).

Kabat-Zinn (1990) recoge siete factores actitudinales fundamentales que el atleta tendrá que aprender para desarrollar una actitud de atención plena, con el objetivo final de afrontar su rendimiento. Dichos factores son: no juzgar, paciencia, mente de principiante, no buscar hacer, confianza, dejar ir/ceder y la aceptación. Por su puesto, los factores o elementos citados anteriormente, mejoraran con el entrenamiento continuo, sin recetas rápidas o mágicas, como si de un entrenamiento de resistencia, fuerza, flexibilidad o veloci- 
dad se tratara, por tanto, es fundamental el compromiso del deportista en cada práctica (Cepeda y Romero, 2014; Segal et al; 2002). Además, los estudios de Slagter, et al. (2007) demuestran que se necesitan al menos 3 meses de experiencia en mindfulness para realizar un uso más eficiente de los recursos atenciones. Otros autores encontraron que tan sólo con 4 días de práctica ya se mejoran las capacidades atenciones de procesamiento viso-espacial, memoria de trabajo y ejecutiva, comparando a los sujetos que lo realizaron con un grupo control (Zeidan, Johnson y diamond, 2010; Goolkasian, 2010).

Centrándonos en el concepto de atención plena, los autores Cepeda y Romero (2014) lo definen como estar en el momento presente y no atender a hechos pasados o anticipar eventos futuros, como ganar o perder. Los deportistas suelen describir la sensación de estar con atención plena, estar conscientes y presentes como estar "en la zona". Entrenar atención plena determina que el atleta tome una perspectiva de sus patrones de respuesta o su manera de afrontar las situaciones de la competición. Por ejemplo, si en un deporte de combate golpean al deportista en el rostro, la activación emocional crece y es probable que la respuesta sea de ataque descontrolado, mientras que, si se ha realizado un completo entrenamiento en mindfulness, el deportista será consciente de su emoción y pensamientos presentes y podrá aceptar el golpe como experiencia propia del combate, dejando ir mediante la respiración las emociones y pensamientos tras el golpe.

Otro ejemplo se encuentra en un estudio realizado por Baltzell, Caraballo, Chipman y Haydn (2014) de carácter cualitativo, para conocer el impacto de un programa de atención plena en mujeres futbolistas de primera división. En este estudio se recogen comentarios de las jugadoras como el siguiente: "creo que soy capaz de enfocar la emoción o ansiedad hacia mi juego en lugar de estar demasiada excitada al principio del partido donde quiero resolver muy rápido", "hacia el final del juego...cuando estaba fatigada y en vez de decir que estoy cansada, aceptaba el dolor y me preparaba para un ataque".

Existe un notable interés en los últimos años por usar las técnicas de mindfulness que acompañen la intervención en Psicología del Deporte, donde ha existido un progreso continuo de desarrollo de las técnicas y programas de intervención con mindfulness en el deporte, aunque no ha sido hasta las últimas dos décadas cuando han comenzado a desarrollarse investigaciones empíricas.

En la actualidad existen dos enfoques empíricos para trabajar el rendimiento de los atletas y que tienen que ver con mindfulness. Por un lado, el programa de Kaufman y Glass (2006) Mindfulness Sport Performance Enhancement
(MSPE), que combina la reducción de estrés mediante mindfulness (MBSR) y la terapia cognitiva basada en mindfulness (MBCT), donde se entrenan habilidades de mindfulness y aceptación del momento. Se trabaja en 4 sesiones con una duración de 2,5 a 3 horas por sesión (Pineau, Glass y Kaufman, 2014).

Por otro, Gardner y Moore (2004 y 2007) realizan Mindfulness-Acceptance (MAC), el cual, combina la terapia cognitiva basada en mindfulness con la terapia de aceptación y compromiso y cambios conductuales. Está estructurado en un programa de 9 a 12 sesiones, con una duración de 45 a 60 minutos por sesión (Palmi y Solé, 2016). Otros autores como Baltzell y Akhtar (2014) crean el Mindfulness Meditation Training for Sport (MMTS), que combina el entrenamiento de mindfulness y de habilidades psicológicas tradicionales, como imágenes o autodiscurso. Se desarrolla en 6 semanas, manteniendo dos reuniones de 30 minutos por semana. También resulta interesante mencionar el entrenamiento de atención plena en la experiencia de flujo (Aherne, Moran y Lonsdale, 2011), consiste en una intervención de 6 semanas realizando ejercicios de meditación, yoga, escaneo corporal y entrenamiento en meditación guiada. Además, Grove, Bernier y Fournier (2013) han desarrollado el primer cuestionario específico para deportistas, Mindfulness Inventory for Sport (MIS) para medir las habilidades de atención plena de los atletas.

Estos programas aplicados en Psicología del Deporte implican un entrenamiento mental y en manejo de emociones que pueden ayudar al deportista a conseguir un rendimiento óptimo y el bienestar psicológico (Carraça et al., 2018). La estructura básica o fases de aplicación del mindfulness según los distintos programas revisados como MAC de Gardner y Moore (2004 y 2007) y MPSE de Kaufman y Glass (2006) se suelen organizar en varios componentes los cuales forman protocolos estructurados o semi-estructurados. Según Mañas, Del águila, Franco, Gil y Gil (2014) el programa MAC intenta conseguir la ejecución óptima del atleta a través de un entrenamiento de cinco fases: (1) psicoeducación, donde se realiza una presentación sobre el funcionamiento del programa y un establecimiento de objetivos; (2) mindfulness, implica entrenar a los atletas en la capacidad de centrar su atención en el momento presente sin juzgar ni evaluar la experiencia y "dejar ir" los pensamientos y emociones no centrados en el "aquí y ahora"; (3) identificación de valores y compromiso, donde se estimula al deportista a vivir una vida según sus valores y no controlada por emociones aisladas. (4) aceptación, se sugiere a los atletas que comparen el coste de vivir acorde a sus propios valores contra los esfuerzos por controlar los estados internos; (5) integración práctica, se practican las anteriores fases y se discuten las posibles dificultades. 
En cambio, el protocolo MSPE de Kaufman y Glass (2006) se lleva a cabo durante 4 semanas, en sesiones de $90 \mathrm{mi}-$ nutos y prácticas en casa. El primer paso es explicarles a los deportistas las características del programa y su utilidad tanto en el deporte como en la vida diaria. En este caso, el programa consta de seis fases: (1) ejercicio del caramelo, donde se les da un caramelo a los deportistas y se les invita a que pongan sus sentidos en él; (2) meditación sentada, se le pide al atleta que focalice la atención en su respiración, en las sensaciones de su cuerpo y en los sonidos de alrededor. Esta fase se comenzara con periodos de 10 minutos y se finalizara con hasta 25 minutos de práctica; (3) body-scan, implica poner la atención en diferentes partes del cuerpo y aceptar cualquier sensación; (4) mindfulness yoga, donde se realizan posturas de yoga y mantenimiento de la atención mientras los atletas están en movimiento; (5) meditación caminando, implica ser conscientes de las sensaciones mientras cambian el ritmo en sus pasos; (6) meditación específlca deportiva, reuniendo los pasos anteriores aplicándolos a un deporte en concreto.

En resumen, en Psicología del Deporte existen múltiples definiciones y aplicaciones eficaces para mejorar el rendimiento y el bienestar de los atletas. La aplicación del mindfulness en contextos deportivos surge como alternativa a las intervenciones cognitivas-conductuales tradicionales donde se enfatiza en el cambio o la eliminación de ciertos eventos internos del deportista. Por el contrario, cuando se interviene con mindfulness se pretende aceptar la experiencia, abandonar la lucha y el control de nuestros eventos internos y adoptar una postura contemplativa. De este modo, surge

DIAGRAMA DE FLUJC
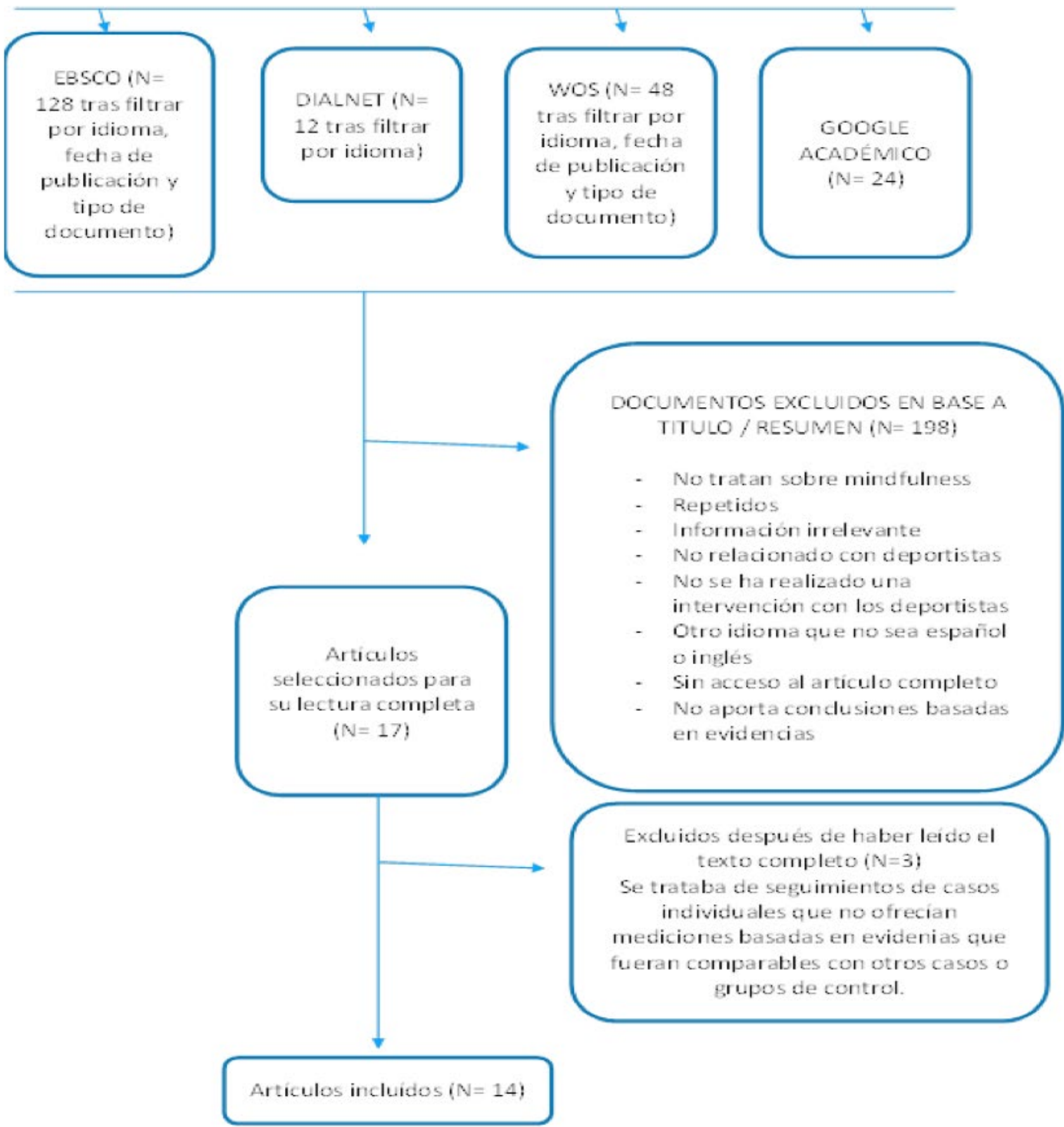

Figura 1. Diagrama de flujo para la revisión de artículo sobre maindfulness y deporte. 
la necesidad de revisar los distintos modelos, protocolos y aplicaciones del mindfulness en Psicología del deporte en busca de las intervenciones que demuestren empíricamente ser eficaces, y así poder compararlas con otros modelos de intervención (Mañas et al., 2014).

Partiendo de estas cuestiones, el objetivo del presente trabajo es realizar una revisión de los trabajos sobre la temática publicados entre los años 2000 al 2018, que han utilizado el mindfulness como estrategia de intervención o el desarrollo de programas de atención plena.

\section{Método}

La revisión de los trabajos se realizó mediante la búsqueda en las bases de datos de EBSCO, Dialnet, WOS y Google académico utilizando las palabras clave: mindfulness, intervención, deporte y rendimiento; y los filtros en las bases de datos: idioma (español e inglés), fecha de publicación (año 2000 al 2018) y tipo de documento (artículo). Destacar que también se han seleccionado artículos anteriores al año 2000 debido a su relevancia para el ámbito de estudio.

Se obtuvieron un total de 212 artículos de los cuales se seleccionaron 14 según los siguientes criterios de inclusión y exclusión (Perestelo, 2013). Como criterios de inclusión se propusieron artículos que traten sobre mindfulness y relacionados con deportes (sports) o deportistas (athletes), que hubieran llevado a cabo una intervención psicológica utilizando las técnicas de mindfulness en un ambiente deportivo, en los idiomas de español e inglés y que garantizaran un acceso gratuito al texto completo.

Los criterios de exclusión eliminaron del estudio todo artículo que no tratase sobre mindfulness o no estuviera relacionado con un contexto deportivo, donde no se haya realizado una intervención psicológica con deportistas, estudios solo de caso único o sin datos concluyentes, trabajos en otro idioma que no fuera español o inglés, artículos que no tuvieran un acceso al texto completo o cuyo acceso se realizara mediante pago. A continuación, se puede encontrar el diagrama de flujo donde se desarrolla de manera esquematizada la metodología seguida (ver Figura 1).

\section{Revisión de artículos}

A continuación, vamos a expresar las diferentes metodologías ordenadas por su mayor o menor estructuración, uso y reconocimiento dentro del ámbito de la Psicología aplicada al deporte. Los trabajos seleccionados se apoyan en una metodología de entrenamiento estructuradas del mindfulness. Algunas de ellas reconocidas y extendidas internacionalmente, otras diseñadas ad hoc según el caso. Las más reconocidas son:

1) MAC: Mindfulness Acceptance-Commitment (MAC) de Gardner y Moore (2004 y 2007). Del Águila el al. (2009) citado en Cangas, Gallego y Navarro (2014) indican textualmente que el programa MAC es una combinación de Mindfulness y técnicas de la Acceptance and Commitment Therapy (ACT) (Hayes et al., 1999); Wilson y Luciano, 2002), que fue diseñado para utilizarse con personas con alto nivel de rendimiento y puede ser usado con atletas. Según los autores, el MAC comparte ciertas similitudes con el concepto de flow (Csikzentmihalyi, 1990) que describe el estado de ejecución ideal en los atletas como una intensificada consciencia al momento presente y concentración en la tarea. El flow también ha sido descrito como la "fusión de la acción y las consciencias" (Csikzentmihalyi, 1990). El programa MAC parece promover el estado de ejecución óptima en atletas y la experiencia de flow. Este programa semi-estructurado está organizado en 5 componentes, incluye: (1) psicoeducación; (2) Mindfulness; (3) identificación de valores y compromiso; (4) aceptación; y (5) integración y práctica (Mañas et al., 2014)

Zhang et al. (2015) estudiaron los efectos del Mindfulness en jugadores noveles de dardos. Los autores quisieron ver el impacto del programa MAC en el rendimiento de jugadores de dardos, así como en los 5 factores de Mindfulness a través de la herramienta Five Facet Mindfulness Questionnaire (FFMQ-SF; Baer, Smith y Hopkins, 2006), y una eventual mejora en la predisposición de lograr el estado de fluidez, a través de las Short Dispositional Flow Sale (Jackson y Eklund, 2004). Se utilizó como muestra un colectivo de 43 estudiantes de primer año de instituto que nunca habían jugado a dardos, y fueron repartidos de manera aleatoria en grupo experimental (22 personas) y grupo de control (21 personas). El grupo entrenado en MAC obtuvo mejoras estadísticamente significativas en las competencias de Mindfulness y en predisposición para el estado de fluidez. Si bien ambos grupos experimentaron una mejora en rendimiento, el grupo entrenado en Mindfulness obtuvo una mayor mejora estadísticamente significativa, respecto al grupo de control. Los resultados se mantuvieron tanto al final del programa, como en un follow up después de dos semanas.

Goodman, Kashdan, Mallard y Schumann (2014), investigaron el impacto de un programa de MAC de Mindfulness y yoga en un equipo de baloncesto de primera división de NCAA. Se quiso analizar el impacto sobre variables como: competencias de Mindfulness, a tráves del Mindful Attention and Awareness Scale (MAAS) (Brown y Ryan, 2003) tolerancia a afecciones negativas, The 25-item Tolerance of Negative Affect States Scale (TNASS) (Bernstein y Brantz, 2012); aceptación, The 17-item Acceptance and Action Question- 
naire Version II (AAQ-II) (Bond et al., 2011) motivación a logros, The Adult Hope Scale (AHS) Snyder et al., (1991); percepción del estrés, The Perceived Stress Scale (PSS) (Cohen, Kamarck y Mermelstein, 1983); compromiso con valores, The Valued Living Questionnaire (VLQ), Wilson, Sandoz, Kitchens y Roberts, (2011); orientación a resultados, The Short Grit Scale (Grit-S) (Duckworth y Quinn, 2009); capacidad de reducir impacto de pensamientos negativos, The Drexel Defusion Scale (DDS) (Forman et al., 2012); estrés perjudicial, The 21-item Depression, Anxiety Stress Scale (DASS-21) (Henry y Crawford, 2005). La intervención duró 5 semanas, con 8 sesiones grupales de 90 minutos, seguidos de una sesión de 60 minutos de Hata Yoga. La muestra fue compuesta por 8 personas y hubo que reclutar un grupo de estudiantes deportistas como grupo de control, compuesto por 13 personas. Los resultados indicaron un incremento en la orientación a objetivos y de las competencias de Mindfulness referido al grupo que realizó el programa. El grupo de control no mostró diferencias en las variables entre el inicio y el final de la investigación. Comparando los grupos tras el programa, no se relevaron diferencias en niveles de ansidedad, estrés y depresión. Se recogió al final del programa un feedback escrito a nivel cualitativo, reflejando el grupo experimental una terminología positiva en comparación con el grupo de control, lo cual hace pensar en una contribución cualitativa del programa hacia actitudes positivas en general.

Otros programa insipirados en el MAC, derivados del mismo, o con influencia de la Terapia de Aceptación y Compromiso: Bernier, Thienot, Codron y Fournier (2009), quisieron analizar el impacto de un programa de entrenamiento en Mindfulness en el rendimiento deportivo y en ciertas habilidades cognitivas asociadas al deporte, relevadas con el Ottawa Mental Skills Assessment Tool-3 (OMSAT-3) (Durand-Bush, Salmela y Green-Demers, 2001). Para ello se sirvieron de una muestra de 7 golfistas de élite (2 mujeres y 5 hombres), de una media de edad de 15 años, con experiencia entre 4 y 10 años, y una práctica de entrenaniemto diario de 3 horas. Se diseñó un programa derivado de la Mindfulness Based Cognitive Therapy (MBCT) (Segal et al., 2002), suplementado por herramientas de Terapia de Aceptación y Compromiso (ACT) desarrollada por Hayes y Strosahl (2004). Los resultados reflejaron mejora significativa respecto al grupo de control, solo en la variable de "activación" que puede asemejarse a la de Atención, que se mide por otros estudios a través de otras herramientas de evaluación competencial de la Atención Plena. Esta intervención hizo uso de un programa clínico para unos beneficiaries que no mostraron necesidades clínicas de intervención. Gardner y Moore (2004, 2007) demostraron que el programa MAC, es efectivo con beneficiarios no clínicos, aunque requiere de ciertas adap- taciones. Los autores de esta investigación consideran su programa similar al programa MAC, pero no realizaron quizá las adaptaciones pertinentes para dirigirlo a usuarios no clinicos. Éste, junto al escaso numero de la muestra, pueden considerarse las principales deficiencias de este studio.

Aukee (2014) quiso investigar un programa de 6 semanas derivado del MAC e integrado de una intervención psicológica de coaching basado en valores, en un equipo femenino de futbolistas de élite. Concretamente analizó el impacto del programa en variables como: aceptación, Acceptance and Action Questionnaire, (AAQ-II) (Bond et al.,2011); percepción del estrés, Perceived Stress Scale, (PSS) (Cohen et al., 1983); autoconfianza, (mediante cuestionario propio); rendimiento, (mediante escala perceptiva propia de factores de rendimiento); bienestar emocional, y social, MHC-SF, Mental Health Continuum Short Form (Lamers, Westerhof, Bohlmeijer, ten Klooster, Keyes, 2011). Todos los participantes recibieron el programa. No hubo grupo de control. Pese a referir las atletas una percepción positiva y de bienestar al final del programa, y pese a mostrarse algunas mejoras en autoconfianza, el trabajo no reflejó resultados significativos.

2) MSPE: Mindful Sport Performance Enhancement (MSPE) de Kaufman y Glass (2006). Del Águila et al. (2009) en Cangas, Gallego y Navarro (2014) indican textualmente que este programa surge del Mindfulness-Based Stress Reduction (MBSR) de Kabat-Zinn (1990) y de la Mindfulness-Based Cognitive Therapy (MBCT) de Segal et al. (2002), así como de otras fuentes relevantes relacionadas con el Mindfulness y el deporte (Gallwey, 1974). Según los autores, el MSPE está estructurado para ser adaptado a cualquier deporte. La versión inicial del MSPE fue diseñado como un protocolo de 4 semanas. Recientemente se ha desarrollado una nueva versión extendida de este programa (De Pedrillo, Kaufman, Glass y Arnokoff, 2009). La versión extendida del MSPE consiste en un protocolo de 6 semanas de duración. Semanalmente tiene lugar una sesión en grupo de 90 minutos. Además, el programa contiene prácticas para ser realizadas en casa diariamente. Los autores se propusieron valorar el impacto del programa en los niveles de rendimiento y de calidad de vida de corredores amateurs de larga distancia. La muestra fue compuesta por 10 hombres y 15 mujeres con edades entre 18 y 55 años que fueron repartidos en dos grupos: uno entrenado en MSPE (13 personas) y otro como grupo de control (12 personas). Para ello se realizó un cuestionario previo y posterior a la intervención que medía la presencia de factores de estrés. Se estudió el impacto del programa en las siguientes variables: Preocupación, Thought Occurrence Questionnaire for Sport (TOQS) (Hatzigeorgiadis y Biddle, 2000); Perfeccionismo, Multidimensional Perfectionism Scale (MPS) (Frost, Marten, Lahart y Rosenblate, 1990); Aten- 
ción, Kentucky Inventory of Mindfulness Skills (KIMS) (Baer, Smith y Allen, 2004); Expectativas de tratamiento, Credibility and Expectations Measure (CEM), Atención, Toronto Mindfulness Scale (TMS) (Lau et al., 2006). También se midió el nivel de rendimiento mediante auto-registros que en los que se indicaba la frecuencia de entrenamiento, la distancia de carrera, los tiempos, y el nivel de satisfacción según una escala Likert de 5 puntos. Los resultados de la investigación indican que la aplicación del programa no aporta una mejora en el rendimiento, si bien ofrece resultados significativos en otras variables de calidad de vida, tales como la reducción del nivel de perfeccionismo, (al que los autores asocian una influencia en los niveles de presión que pueden afectar al rendimiento), y competencias relacionadas con la capacidad de estar atentos al presente (Mindfulness) y el estado de vigilia (Awareness). Los resultados de una regresión estadística revelaron que las altas expectativas sobre el programa influyen en la reducción del nivel de perfeccionismo, y que cuanto más elevada es la presencia de fuentes de estrés con las que el deportista está en contacto, mayor es la reducción de los niveles de preocupación.

Otro estudio de Thompson, Kaufman, De Petrillo, Glass y Arnkoff, (2011), pretendió analizar los efectos a largo plazo del programa MSPE. Se realizó un follow up durante un año a arqueros, golfistas y corredores de larga distancia para observar los efectos de MSPE en variables como las competencias de Mindfulness a través del Kentucky Inventory of Mindfulness Skills, (KIMS) (Baer et al., 2004). Además, para los golfistas y arqueros se añadió la medición de: pensamientos irrelevantes, a través del Thought Occurrence Questionnaire for Sport (TOQS) (Hatzigeorgiadis y Biddle, 2000); ansiedad deportiva, Sport Anxiety Scale (SAS) (Smith, Smoll y Schutz, 1990); autoconfianza en deporte, Carolina Sport Confdence Inventory (CSCI) (Manzo, Silva y Mink, 2001); y estado de fluidez, Dispositional Flow Scale-2 (DFS2), (Jackson y Eklund, 2002). Para los corredores se aplicó la medición de perfeccionismo, Multidimensional Perfectionism Scale (MPS) (Frost et al., 1990). Además, se añadieron una serie de cuestionarios que se prepararon ad hoc para el estudio, con el fin de tener en cuenta variables ambientales que podrían afectar a los deportistas: Archery Questionnaire (AQ) y Golf Questionnaire (GQ). The 8-item AQ and the 10item GQ. La muestra estuvo compuesta por 4 arqueros, 8 golfistas y 13 corredores de larga distancia. La relevación de los datos fue acompañada de registros sobre el nivel de rendimiento que indicaban frecuencia de entrenamiento, resultados y así como sus sentimientos acerca de su rendimiento. Los resultados indicaron incrementos significativos de las competencias de Mindfulness, y las habilidades como la vigilia (Awareness) así como reducción significativa de preo- cupaciones y pensamientos irrelevantes. Los corredores de larga distancia desarrollaron un incremento significativo de su rendimiento en los tiempos por milla, con una correlación positiva entre rendimiento y variables de tratamiento.

3) Mindfulness Meditation Therapy (MMT) de John, Verma y Khanna (2011). El método dura 4 semanas, con sesiones de 20 minutos en grupos de 8 personas entre las 8 y 10 de la mañana, 6 días a la semana. El objetivo del estudio era valorar el impacto del MMT en los indicadores de cortisol salivar, considerado un predictor fiable de los niveles de estrés. Se realizó un pre, post y follow up con una muestra de 26 tiradores de pistola profesionales, entre 25 y 33 años de edad. 48 fueron asignados aleatoriamente un grupo de control. La toma de muestras de saliva se realizó el día 1 el 29 y el 36 del programa. También se midió el nivel de rendimiento mediante una prueba el día antes de la competición pre definida (John et al., 2011). El resultado indicó diferencias significativas respecto al grupo de control, tanto en nivel de cortisol, como en rendimiento deportivo: se concluye que 4 semanas de MMT reducen el nivel de cortisol respecto al grupo de control. Sobre el estudio hay que añadir que se les pidió a los participantes no consumir estimulantes como cafeína, te, etc. Este elemento pudo contribuir en los resultados tan significativos, implicando cierta dificultad en hallar cuál de los dos factores (el programa o la abstinencia de estimulantes) pudo tener más impacto en ello.

4) $\mathrm{MiBCT}(\mathrm{Mi}=$ Mindfulness Integrated, Based Cognitive Therapy) de Scott-Hamilton, Schutte y Brown (2016). Consiste en un programa de 8 semanas con un taller semanal, ejercicios para casa guiados con un CD, y sesiones en grupo de entrenamiento en bicicleta estática para aplicar los conceptos de Mindfulness a la actividad deportiva. Es un Programa especialmente diseñado para ciclistas. Tiene 8 semanas de duración e incorporaba ejercicios de pedaleo consciente. Se quiso valorar el impacto del programa en las siguientes variables con sus respectivas herramientas de medida: estado de flujo, Dispositional Flow Sale II (DFS-2) (Jackson y Eklund, 2002); ansiedad de competición, Sport Anxiety Scale (SAS) (Smith, et al., 1990); ansiedad en deporte, Sport Anxiety Scale 2 (SAS-2) (Smith, Smoll, Cumming y Grossbard, 2006); competencias de Mindfulness, Five Facet Mindfulness Questionnaire (FFMQ-SF) (Baer et al., 2006); estilo atribucional en deporte, Sport Atribution Style Scale (SASS) (Hanrahan, Grove y Hattie, 1989). Para ello se contó con 27 ciclistas en grupo experimental y 20 en grupo de control. Fueron asignados aleatoriamente a cada grupo. Hubo mediciones pre y post intervención. El estudio arrojó resultados significativos en las tres variables medidas (atención plena, fluidez, mejora de la ansiedad deportiva y mejora del pesimismo). Se registró co-relación entre mejora del Mindfulness y mejora del flujo. 
No hubo resultados significativos entre pre-test y pos-test en cuanto a pesimismo en el grupo de intervención, pero si lo hubo en cuanto a ansiedad, Mindfulness y flujo. Además, los ciclistas del grupo de intervención no mostraron una mayor frecuencia de conectar con la sensación de fluidez, respecto al grupo de control. Es importante señalar que el estudio no aclara si se realizó un ciego entre diseñadores del programa, monitores, e investigadores.

5) MMTS Mindfulness Meditation Training for Sport (MMTS) de Baltzell y Aktar (2014). Es un programa de 6 semanas de duración que consiste en dos encuentros de 30 minutos cada semana y la integración del entrenamiento en Mindfulness con tradicionales entrenamientos de habilidades psicológicas. Las discusiones y ejercicios se centraron en enseñar una consciencia abierta y receptiva, el uso de afirmaciones positivas, concentración y tácticas para gestionar los estados mentales negativos. Además, se les invitaba a los atletas a que practicaran en su vida diaria. El studio se proponía medir el efecto del programa MMTS en bienestar psicológico The Psychological Well-Being Scale (PWBS): The PWBS (Ryff y keyes, 1995). Alerta (Awareness), Mindful Attention Awareness Scale (MAAS): The 15-item MAAS (Brown y Ryan, 2003); estado de ánimo positivo y negativo, The Positive Affect, Negative Affect Schedule (PANAS): The PANAS (Watson, Clark, y Tellegen, 1988), satisfacción de vida, The Satisfaction with Life Scale (SWLS): The SWLS (Diener, Emmons, Larson y Griffin, 1985). Para ello se aplicó el programa a un colectivo de 42 atletas femeninas de fútbol y remo, del noreste de Estados Unidos, en edad de instituto, que fueron repartidas en dos grupos: experimental (19 personas del equipo de fútbol) y de control (23 personas del equipo de remo). Los resultados mostraron que los atletas que recibieron el programa MMTS mostraron un incremento significativo en los niveles de Mindfulness en comparación con los del grupo de control, quienes mostraron un incremento de emociones y sentimientos negativos, cosa que no ocurrió en el grupo entrenado en MMTS.

El resto de programas contemplados en las publicaciones seleccionadas, se diseñaron ad hoc y no responden a protocolos estándar. Para evitar entrar en el detalle de cada uno, se ofrece una síntesis de los principales hallazgos y conclusiones de cada artículo:

Atchley (2011). An Examination of the Effects of Mindfulness and Task-Relevant Attentional Focus on Running Performance. La investigación quiso analizar el impacto de la práctica del Mindfulness sobre variables como: foco atencional, Measure of attentional focus (MAF) (Wininger y Gieske, 2010); vigilia, Mindful attention awareness scale (MAAS); percepción de esfuerzo, Borg ratings of perceived exertion (RPE) scale (Borg, 1998); distress, Distress Scale
(Brewer, Van Raalte y Linder,1996); percepción del cambio de estado, Marcus, Selbey, Niaura y Rossi (1992), y rendimiento deportivo. El estudio se realizó con una muestra de estudiantes no corredores, con una media de edad de 19 años repartidos en grupo experimental (41 personas) y grupo de control (42 personas), de las cuales 41 eran hombres y 42 mujeres, entrenados para practicar un ejercicio de atención plena de 30 minutos, tres veces por semana. Las mediciones de rendimiento se realizaron sobre los tiempos de carrera en la distancia de dos millas. No se encontraron diferencias significativas en rendimiento de los dos grupos. Tampoco se observaron diferencias significativas en cuanto a la capacidad de foco atencional. No se encontraron mejoras en cuanto a niveles de vigilia en el grupo entrenado, entre la relevación de los datos al principio y al final del programa. El entrenamiento en atención sobre sensaciones corporales durante el ejercicio, para el grupo entrenado en la tarea de Mindfulness mostró mayores niveles de distress, aunque no significativos. Los investigadores consideran que los escasos resultados del estudio se pueden atribuir a que las personas entrenadas no eran deportistas profesionales, No estarán expuestos a altas fuentes de estrés y su práctica no implicaba la necesidad profesional de mejorar sus marcas de cara a las competiciones: este elemento pudo sesgar la investigación y hace pensar en la necesidad de realizar estudios con deportistas profesionales.

Thakur y Mahesh (2016) quisieron observar los resultados de un programa de entrenamiento en meditación Mindfulness de duración de seis semanas, sobre el nivel de rendimiento de jugadores de baloncesto, medido a través de la ratio entre aciertos y tiros de campo en entrenamiento, desde distancias variadas. Para ello se contó con una muestra de 30 varones divididos en dos grupos de 15 personas: el grupo de control y el grupo experimental que realizó el programa. No se obtuvieron resultados significativos. Cabe destacar que no se midió el rendimiento en competición, sino en tiros de campo en entrenamiento, lo cual pudo sesgar los resultados finales por no estar sometidos los jugadores a los niveles de estrés habituales de competición.

MacDonald, Oprescuc y Keanb (2018) An evaluation of the e ects of Mindfulness training from the perspectives of wheelchair basketball players. Se quiso investigar el impacto de un programa de entrenamiento en Mindfulness de 8 semanas, sobre niveles de calidad de vida de deportistas en silla de ruedas. El programa incluye unos ejercicios 5 días a la semana, asistidos mediante la aplicación de móvil Smiling Mind que se adquiere mediante App store de Iphone o Play Store de Google. Para ello se utilizó una muestra de 8 jugadores de baloncesto en silla de ruedas. Se trataba de 6 hombres y 2 mujeres de la misma categoría, con promedio 
de edad de 29 años, con menos de 2 años de experiencia competitiva, y al menos 15 horas de entrenamiento semanal. La medición de variables se realizó mediante relatos auto-perceptivos. Si bien al finalizar el programa los relatos mostraban una mejora percibida en concentración, calidad de sueño, regulación de ritmo cardíaco, y tasa de fallos en tiros de campo, no se aportan mediciones objetivas que respalden estas impresiones, que solamente fueron auto-relatadas por los 8 participantes.

MacDonald y Minahan (2018). Mindfulness training attenuates the increase in salivary cortisol concentration associated with competition in highly trained wheelchairbasketball players. Estos autores analizaron el impacto de un programa de 8 semanas de Mindfulness en los niveles de cortisol y de la imunoglobina A en la saliva, considerando estos indicadores como fiables predictores de los niveles de estrés. Para ello se utilizó una muestra de 16 jugadores de baloncesto en silla de ruedas repartidos aleatoriamente en dos grupos de 8 personas (grupo experimental de 5 hombres y 3 mujeres, y grupo de control, de 6 hombres y 3 mujeres). Se trataba de jugadores de la misma categoría, con promedio de edad de 27 años, con menos de 2 años de experiencia competitiva, y al menos 15 horas de entrenamiento semanal. Si bien no se encontraron resultados significativos en cuando a los niveles de imunoglobina A entre los dos grupos, se halló una diferencia significativa entre grupo experimental y grupo de control referida a una reducción del nivel de cortisol en el grupo experimental, durante la época de competición, permitiendo concluir que la práctica de la atención plena contribuye a una regulación del estrés, por considerarse el cortisol un fiable predictor del mismo.

Franco (2009) expone la modificación de los niveles de burnout y de personalidad resistente en un grupo de deportistas a través de un programa de conciencia plena (Mindfulness). El autor se propuso analizar los efectos de un programa de Mindfulness sobre los niveles de burnout, medido con el Inventario de Burnout en Deportistas (IBD) de Garcés de los Fayos (1999), y de personalidad resistente, evaluado con la Personal Views Survey (PVS) del Hardiness Institute (1985). Se trata de un programa de 10 semanas con una sesión semanal de hora y media y la práctica de un ejercicio diario de meditación de 40 minutos Para ello, contó con la participación de un grupo experimental y un grupo control. Un total de 41 deportistas federados que estaban participando en competiciones oficiales a nivel provincial, regional o nacional, y que se encontraban realizando estudios en diversas titulaciones de la Universidad de Almería (España). Las edades de los participantes oscilaban entre los 18 y los 32 años. La asignación se realizó de manera aleatoria, controlando un adecuado reparto de los deportistas por sexo y de- porte de proveniencia (atletismo, natación, tenis, baloncesto, balonmano, fútbol y vóley). Los grupos fueron sometidos a tres mediciones (pretest, postest, y follow up a distancia de 5 meses). Los análisis realizados muestran la existencia de diferencias estadísticamente significativas entre grupo experimental y grupo de control. Se obtuvo una mejora significativa en el grupo experimental respecto al grupo control en las tres dimensiones que evalúan la personalidad resistente (control, compromiso y reto), y en dos de las tres dimensiones que componen el burnout deportivo (escasa realización personal y agotamiento emocional). Dichas diferencias se mantuvieron cinco meses de la finalización de la intervención la intervención. El estudio no menciona si el programa de Mindfulness estaba integrado como complemento de algún tipo de intervención psicológica. Del mismo modo que no aclara si hubo metodología ciega entre quien creó la metodología, quien impartió el programa, y quien investigó

\section{Discusión}

El objetivo de este trabajo era realizar un análisis de las publicaciones en las dos últimas décadas sobre la intervención psicológica en deportistas usando técnicas de Mindfulness o creación de nuevos programas y herramientas para conseguir "atención plena".

Los resultados de las investigaciones analizadas muestran que a pesar de que existen distintos tipos de intervención con Mindfulness en deportistas, los programas Mindfulness-Acceptance (MAC) (Gardner y Moore, 2007) y Mindfulness Sport Performance Enhancement (MSPE) (Kaufman y Glass, 2006) son los modelos que ofrecen un mayor número de publicaciones basadas en evidencia.

No obstante, todos los programas de intervención se caracterizan por unos criterios comunes: duración entre 4 y 10 semanas, una sesión presencial semanal de alrededor de 90 minutos, y una práctica en cada (mediante registros, app, o tutoriales en audio) de entre 40 y 60 minutos, con una frecuencia que oscila entre lo diario y los tres días por semana.

Con respecto a las muestras en los distintos trabajos no varían en género, pero sí en número de personas, siendo habitual contar con muestras muy reducidas en todos los programas, entre 7 y 40 personas. En los estudios analizados en el apartado anterior las muestras por encima de 40 personas son casi inexistentes, dato que invita a necesidad de mayores muestras para ampliar la fiabilidad de las conclusiones que ofrecen.

Con respecto a los deportes elegidos para realizar, no se refleja una predominancia en la aplicación en algún deporte en especial. Los deportistas que más se han beneficiado de 
los trabajos encontrados son los que pertenecen a deportes de individuales.

Algunas publicaciones que no logran resultados significativos, no cuentan con deportistas profesionales o de alto rendimiento, lo cual puede representar una limitación por no estar expuestas estas muestras a altos niveles de estrés: Atchley (2011) y Zhang et al. (2015). Sin embargo, todos los estudios que se aplicaron con deportistas profesionales y con metodologías de grupo de control, mostraron resultados significativos (Baltzell y Aktar, 2014; Bernier et al., 2009; De Petrillo Kaufman, Glass y Arnokoff, 2009; Franco, 2009; Goodman et al., 2014; John, Verma, Khanna, 2011; Scott-Hamilton et al., 2016; Thompson, Kaufman, De Petrillo, Glass, Arnkoff, 2011). No hay que olvidar que el Mindfulness suele asociarse a intervenciones relacionadas con el estrés y que la aplicación a personas que no necesariamente experimenten ciertos niveles de estrés, puede mermar la significatividad de los resultados.

En cuanto al impacto del Mindfulness en determinadas variables psicológicas, los estudios arrojan poca claridad en la evidencia de un impacto positivo en la regulación de los niveles de estrés: por un lado, hay estudios que avalan esta tendencia (MacDonald y Minahan, 2018), que contrastan con los resultados de otros estudios (Goodman et al., 2014). Al parecer, si nos guiamos por indicadores bioquímicos como el cortisol, los resultados avalan esta hipótesis, pero si nos basamos en las valoraciones cualitativas de herramientas psicológicas como los cuestionarios autosuministrados, los resultados contrastan: Atchley (2011); Goodman et al. (2014), no relevan mejoras, mientras que la investigación de Scott-Hamilton et al. (2016), devuelve resultados significativos sobre mejora de ansiedad.

Donde no parece haber dudas es en el impacto del Mindfulness en la mejora de competencias cognitivas atencionales (Mindfulness) y de vigilia (awareness) 7 de las 8 investigaciones que median el impacto en estas variables, arrojan resultados significativos (Baltzell y Aktar, 2014; Bernier et al., 2009; De Pedrillo et al., 2009; Goodman et al., 2014; Scott-Hamilton et al., 2016; Thompson et al., 2011; Zhang, et al., 2015).

Si parece que el Mindfulness ayuda a generar mayor compromiso y orientación a objetivos, ya que los dos estudios que se propusieron medir estas variables, arrojaron resultados significativos (Franco, 2009; Goodman et al., 2014).

En cuanto a la relación entre Mindfulness y rendimiento deportivo, se presentan conclusiones contrastantes: de los 6 estudios que medían este impacto, 3 no encontraron resultados significativos (Atchley, 2011; De Pedrillo et al., 2009; Thakur y Mahesh, 2016), mientras la otra mitad confirma esta hipótesis (John et al., 2011; Thompson et al., 2011; Zhang, et al. 2015).
Destacamos la gran recurrencia de algunos estudios a relatos personales sobre las experiencias con estos programas: (Aukee, 2014; Goodman et al., 2014; MacDonald et al., 2018; Scott-Hamilton et al., 2016) ponen en evidencia como la práctica del Mindfulness genera actitudes positivas y mejoras en las calidades de vida que los beneficiarios afirman experimentar, aún sin ello poderse reflejar en de manera significativa en las variables que los investigadores monitorizaban.

Este último dato induce enfocar para el futuro la investigación en Mindfulness asociándola a variables de Psicología Positiva y de Crecimiento Personal (Optimismo Disposicional, Engagement, Autoeficacia, Felicidad, etc.), en lugar de las variables clásicas de tipo clínico.

Con respecto a los diseños de investigación, la mayoría de los diseños de los estudios son de tipo longitudinal con Pre-Test y Post-Test, con grupo control, aleatorios y de intervención única con cada deportista. Solo el estudio de Aukee (2014) no contó con grupo de control. Se deduce que para investigar Mindfulness en deporte, es importante realizar estudios longitudinales (pre-test y post-test), y diseños con grupos de control.

En otra revisión sistemática realizada por Palmi y Solé (2016) se detalla que las mayores limitaciones encontradas en las investigaciones sobre Mindfulness en Deportes tienen que ver con la escasa estandarización de los protocolos de intervención y con el exceso de variedad y adaptación de las intervenciones a los casos aislados. Salvando las investigaciones sobre los programas MAC, MSPE, el resto de los programas son muy variados.

Por otro lado, hay quienes sostienen que, para mejorar el impacto de los programas de intervención en psicología del deporte, es necesario proceder a una adaptación individualizada en lugar de colectiva, (Martin y Toogood, 1997). Esta tendencia va justo en antítesis con la crítica de Palmi y Solé (2016), y merece la pena ser considerada: adaptar e individualizar las metodologías puede que sea clave para obtener resultados más eficaces, aunque es posible que una de las principales consecuencias sea que el impedimento en disponer de grandes muestras para validar los mismos programas de intervención, y hallar resultados significativos a la vez.

Como aportación a futuros trabajos similares, será interesante usar las mismas herramientas de medición y analizar las mismas variables que reflejan los estudios analizados en esta revisión, con el fin de comparar los resultados entre sí.

En conclusión, las intervenciones con Mindfulness en el deporte parecen estar a la orden del día. Se necesitan más trabajos que aporten mayores muestras y objetividad a la intervención. Desde una perspectiva integradora podemos 
decir que este tipo de técnicas podrían encajar perfectamente en programas de intervención cognitivo-conductuales o de otro tipo. Con todo ello, las técnicas de Mindfulness parecen dar resultados esperanzadores en la intervención con deportistas.

Entre las causas que parecen abocar a la dificultad de establecer y testar protocolos estándar de intervención, ha de mencionarse el carácter variado de las necesidades que inducen a la aplicación del Mindfulness, así como el carácter cambiante de la evolución de los destinatarios a lo largo del proceso de intervención, lo cual puede implicar el recurso, por parte del técnico, a cambios repentinos frente al plan de trabajo establecido, en pro de un mayor provecho de los beneficiarios, y de una adaptación a sus circunstancias también muy sujetas a la evolución de sus resultados en entrenamiento y competición. No hay que olvidar que la intervención tiene un fin último, frente al mero testeo de una técnica en ambiente controlado: ese fin último es el beneficio del usuario. Esta reflexión pone de manifiesto la necesidad de analizar la eventual conveniencia o no de investigar sobre metodologías y programas estructurados de intervención, ante el hecho de investigar solamente sobre los resultados de aplicación de simples técnicas y ejercicios específicos.

Existe la necesidad de que los nuevos estudios de intervención se esfuercen en utilizar herramientas metodológicas que aumenten la fiabilidad y validez de sus investigaciones (grupos de control, muestras de mayor tamaño, validez interna). Sería conveniente aumentar la objetivad en los estudios, tanto en sus herramientas de evaluación (test, cuestionarios, variables fisiológicas) como en sus intervenciones (registros, análisis de datos, estrategias probadas). Por el contrario, existen algunos estudios como el MAC y el MPSE con un protocolo a seguir, los cuales aportan mayor rigor a las intervenciones, aunque el mayor número de publicaciones no ha de atribuirse a una mayor difusión y aceptación de estas metodologías, sino a una mayor producción investigadora de los autores.

Los estudios examinados, parecen otorgar roles distintos al Mindfulness, posicionándolo en algunos titulares como una metodología de intervención, y considerándolo, en otros estudios como un complemento a la intervención psicológica, o incluso como alternativa (Thompson et al; 2011). Hemos de señalar que por Mindfulness se entiende como una práctica meditativa, que atiende a un propósito muy preciso, "prestar atención al presente, momento por momento, siguiendo el procedimiento y la intención de no juzgar lo que está sucediendo" (Kabat-Zinn, 2003).

El Mindfulness se presenta en los programas más estructurados, como una técnica de apoyo a la intervención psicológica, consistente en ejercicios que imparten profesionales o expertos en la práctica del Mindfulness: en las investigaciones consideradas, no se hace referencia explícita a que los maestros o entrenadores en ejercicios de Mindfulness sean psicólogos, sino más bien expertos en la práctica de esa disciplina meditativa. No queda clara la profesión de los agentes que intervienen en la aplicación de estas prácticas. En los casos y publicaciones analizadas, no se menciona la titulación profesional y proveniencia académica de los monitores que realizaban los programas. Esto debe de prevenirnos ante la falacia de reduccionista de considerar el Mindfulness como intervención psicológica per sé, sino admitirla como un conjunto de prácticas que, en momentos dados, según las necesidades u objetivos, pueden ser usados como complemento a intervenciones psicológicas: y que han de ser los psicólogos los profesionales en cuyas manos residen las decisiones y la responsabilidad reside la responsabilidad de prescribir o no este tipo de prácticas.

\section{Aplicaciones prácticas}

Tras el análisis de los distintos artículos valorados, podemos concluir que para las aplicaciones del Mindfulness por parte de psicólogos en ámbito deportivo, hemos de considerar varios aspectos:

1. Los ámbitos y las circunstancias que hacen susceptible el recurso a esta práctica;

2. Los destinatarios de las intervenciones;

3. El rol o papel de los profesionales que intervienen en ello, el formato de los programas y las técnicas.

Referidos a los ámbitos y circunstancias de aplicación un psicólogo puede recurrir al mindfulness ante las siguientes necesidades: entrenamiento cognitivo de la atención y concentración para el alto rendimiento; regulación del estrés; educación en reconocimiento y gestión de las emociones; gestión de variables psicofisiológicas a través del entrenamiento propioceptivo; como complemento a intervenciones orientadas a la recuperación de lesiones; gestión y aceptación del fracaso; como formación psico-pedagógica en general para escuelas de deporte y escuelas de padres.

Los destinatarios de estos ámbitos de intervención pueden ser variados: un psicólgo que se plantee aplicar el mindafulness puede encontrar como destinatario de sus servicios a los siguientes colectivos: los deportistas de alto rendimiento (para mejorar control emocional, concentración y foco) y deportistas amateurs (en los que se concentra la totalidad de los estudios). Si bien no se ha en- 
contrado estudios destinados a otros agentes que operan en ámbito deportivo, nuestra reflexión se extiende a contemplar como destinatarios también al personal auxiliar y staff técnico de departamentos de salud (Psicólogos, Fisioterapeutas, Preparadores Físicos, Nutricionistas y Médicos) que tenga interés en integrar esta práctica como una herramienta complementaria de su intervención. Atendiendo además a los beneficios que arrojan algunos estudios, consideramos que un psicólogo puede además recurrir al mindfulness para trabajar con entrenadores, educadores, árbitros y jueces. Para entrenadores, árbitros y jueces, el principal beneficio de la práctica puede residir en un cultivo de su bienestar ante situaciones de estrés provocadas por la constante toma de decisiones y el enfoque habitual sobre los resultados que puede provocar un deterioro a nivel nervioso, cognitivo o emocional, además de fomentar la capacidad de compasión y amabilidad que esta práctica contribuye a desarrollar.

Tras la discusión generada entre los responsables de esta publicación, se llegó a la conclusión que para una adecuada y eficaz aplicación de la técnica por parte de psicólogos sería conveniente partir de la necesidad del beneficiario y no del diseño premeditado del programa. En este sentido son los programas que han de adaptarse al cliente y no lo contrario. Quizá haya alguna alternativa para lograr estandarizar la técnica e ir en favor de la comodidad y facilidad de aplicación que proporcionan de los programas predefinidos, sin por ello caer en la no consideración de las necesidades de cada caso en cuestión.

Tras analizar y reflexionar sobre las publicaciones analizadas, según los autores de esta publicación, un psicólogo que quiera intervenir en ámbito deportivo con mindfulness deberá considerar los siguientes pasos en los que podrían participar distintos profesionales (ver Figura 2).
1. Atención al usuario y estudio de las necesidades y diagnóstico.

2. Toma de decisión sobre técnica, suministro, duración y frecuencia.

3. Diseño del programa (tipo de ejercicios, duración y frecuencia).

4. Establecimiento de Indicadores de medición y elección de las herramientas apropiadas.

5. Aplicación de las técnicas.

6. Medición continúa de los resultados con las herramientas seleccionadas e información a profesionales.

7. Ajustes del programa.

8. Análisis de resultados y feedback al beneficiario.

Un psicólogo que recurra a aplicar programas de mindfulness tendrá que diferenciar la intervención individual de la colectiva, puesto que el mindfulness puede ayudar a objetivos diferentes. Tendrá que considerar la diferencia sustancial entre estas tres circunstancias de aplicación: equipos, grupos e individuos aislados, puesto los objetivos perseguibles y la contribución de la técnica podría afectar a variables muy distintas.

Finalmente cabe mencionar que existe un territorio de intervención todavía inexplorado para psicólogos que consiste en el uso de la tecnología para la aplicación y medición de los resultados en deporte, así como la obtención del Big Data derivado, que podría contribuir a una mayor precisión en el ámbito de investigación. No se han encontrado estudios que tengan en cuenta este ámbito que representa, posiblemente, el mayor reto y oportunidad tanto para el desarrollo de programas, como la validación estadística de los mismos en base a la gran cantidad de datos que pueda aportar el recurso a esta nueva tecnología.

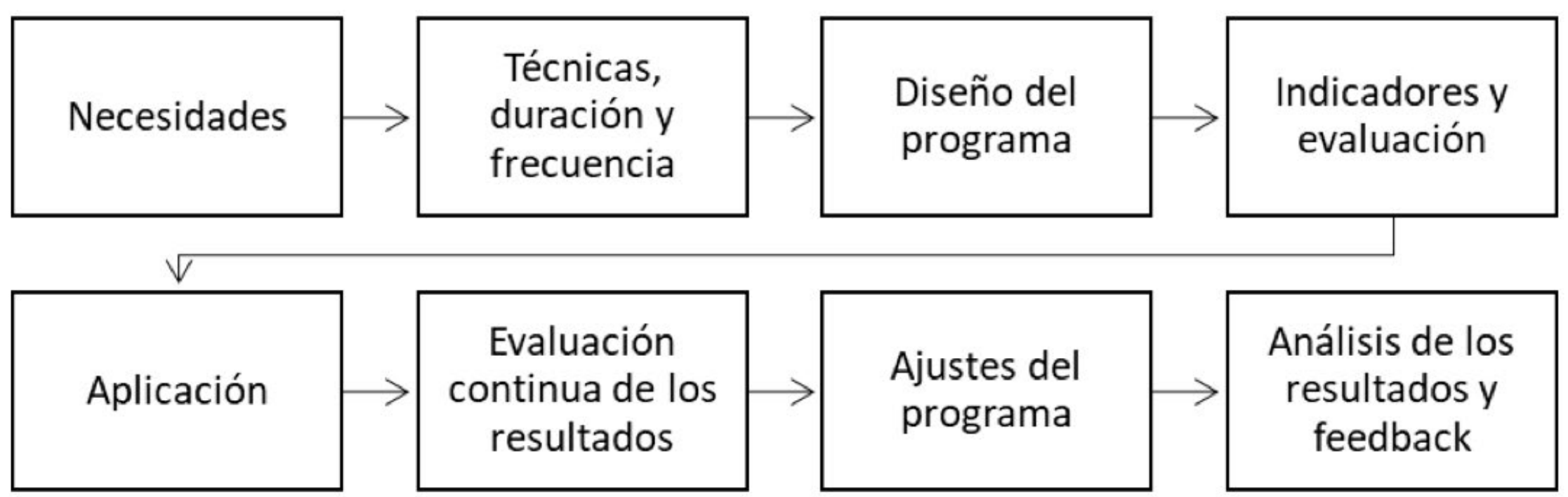

Figura 2. Procedimiento para la aplicación de un programa de mindfulness. 


\section{Referencias}

Aherne, C., Moran, A. y Lonsdale, C. (2011). The effects of mindfulness training on athletes' flow: an initial investigation. The Sport Psychologist, 25, 177-189. https://doi.org/10.1123/tsp.25.2.177

Atchley, A. R. (2011). An Examination of the Effects of Mindfulness and Task-Relevant Attentional Focus on Running Performance. (Tesis de Maestría) Department of Psychology Western Kentucky University, Kentucky.

Aukee, H. (2014). Acceptance, mindfulness and value-based psychological coaching for elite female floorball players. (Tesis de Maestría) University of Jyväskylä Department of Psychology. Recuperado de https://jyx.jyu.fi/dspace/handle/123456789/44645

Baer, R. A., Smith, G. T. y Allen, K. B. (2004). Assessment of Mindfulness by Self Report: The Kentucky Inventory of Mindfulness Skills. Assessment, 11, 191-206. https://doi. org/10.1177/1073191104268029

Baer, R. A., Smith, G .T. y Hopkins, J. (2006). Using self report assessment methods to explore facets of mindfulness. Assessment, 13, 27-45. https://doi.org/10.1177/1073191105283504

Baltzell, A. y Akhtar, V. (2014). Mindfulness meditation training for sport (MMTS) intervention: Impact of MMTS with division I female athletes. The Journal of Happiness \& Well-Being, 2, 160-173.

Baltzell, A., Caraballo, N., Chipman, K. y Hayden, L. (2014). A Qualitative Study of the MindfulnessMeditation Training for Sport: Division I Female Soccer Players'. Experience Journal of Clinical Sport Psychology, 8, 119-141.

Bernier, M., Thienot, E., Codron, R. y Fournier, J. F. (2009). Mindfulness y Acceptance Approaches in Sport Performance. Journal of Clinical Sports Psychology. Human Kinetics, 4, 320-333. https:// doi.org/10.1123/jcsp.3.4.320

Bernstein, A. y Brantz, H. (2012). Tolerance of Negative Affective States (TNAS): Development and evaluation of a novel construct and measure. Cognitive Therapy and Research, 37, 421433. https://doi.org/10.1007/s10608-012-9471-6

Bishop, S., Lau, M., Shapiro, S., Carlson, L., Anderson, N., Carmody, J., ... Devins, G. (2006). Mindfulness: A proposed operational definition. Clinical Psychology. Science and Practice, 10, 230241. https://doi.org/10.1093/clipsy.bph077

Bond, F. W., Hayes, S. C., Baer, R. A., Carpenter, K. M., Guenole, N., Orcutt, H. K., ... Zettle, R. D. (2011). Preliminary psychometric properties of the Acceptance and Action Questionnaire-II: A revised measure of psychological inflexibility and experiential avoidance. Behavior Therapy, 42, 676-688. https://doi.org/10.1016/j.beth.2011.03.007

Borg, G. (1998). Borg's Perceived Exertion and Pain Scales. Champaign, IL, Estados Unidos: Human Kinetics.

Brewer, B. W., Van Raalte, J. L y Linder, D. E. (1996). Attentional Focus and Endurance Performance. Applied Research in Coaching and Athletics Annual, 17, 1-14.

Brown, K. W. y Ryan, R. M. (2003). The Benefits of Being Present: Mindfulness and Its Role in Psychological Well-Being. Journal of Personality and Social Psychology, 84, 822-848. https://doi. org/10.1037/0022-3514.84.4.822

Cangas, A. J., Gallego, J. y Navarro, N. (Eds.) (2014). Actividad física y deporte adaptado. Granada, España: Alborán Editores.
Carraça, B., Serpa, S., Palmi, J. y Rosado, A. (2018). Optimización del Rendimiento Deportivo en los Atletas de Elite: Las Intervenciones Basadas en la Atención Plena. Cuadernos de Psicología del Deporte, 18(2), 79-109.

Cepeda, V. M. y Romero, A. E. (2014). El deportista y la atención plena. Liminales. Escritos sobre Psicología y Sociedad, 5(1), 173187.

Cohen, S., Kamarck, T y Mermelstein, R. (1983). A global measure of perceived stress. Journal of Health and Social Behavior, 24, 385-396. https://doi.org/10.2307/2136404

De Petrillo, L. A., Kaufman, K. A., Glass, C. R. y Arnkoff, D. B. (2009). Mindfulness for Long Distance Runners: An Open Trial Using Mindful Sport Performance Enhancement (MSPE). Journal of Clinical Sports Psychology, 4, 357-376. https://doi.org/10.1123/ jcsp. 3.4.357

Del Águila, J., Mañas I., Franco, C., Gil C. y Montoya, M. (2009). Programas Basados en Mindfulness para Atletas. En A. J. Cangas, J. Gallego y N. Navarro (Eds.), Actividad física y deporte adaptado, (vol. 2, pp. 149-158). Granada, España: Alborán Editores.

Diener, E., Emmons, R. A., Larson, R. J. y Griffin, S. (1985). The satisfaction with life scale. Journal of Personality Assessment, 49, 71-75. https://doi.org/10.1207/s15327752jpa4901_13

Duckworth, A. L. y Quinn, P. D. (2009). Development and validation of the Short Grit Scale (Grit-S). Journal of Personality Assessment, 91, 166-174. https://doi.org/10.1080/00223890802634290

Durand-Bush, N., Salmela, J. H. y Green-Demers, I. (2001). The Ottawa Mental Skills Assessment Tool (OMSAT-3). The Sport Psychologist, 15, 1-19. https://doi.org/10.1123/tsp.15.1.1

Forman, E., Herbert, J., Juarascio, A., Yeomans, P., Zebell, J., Goetter, E. y Moitra, E. (2012). The Drexel Defusion scale: A new measure of experiential distancing. Journal of Contextual Behavioral Science, 1, 55-65. https://doi.org/10.1016/j.jcbs.2012.09.001

Franco, C. (2009). Modificación de los niveles de burnout y de personalidad resistente en un grupo de deportistas a través de un programa de conciencia plena (mindfulness). Anuario de Psicología, 40, 377-390.

Frost, R. O., Marten, P., Lahart, C. y Rosenblate, R. (1990). The dimensions of perfectionism. Cognitive Therapy and Research, 14, 449-468. https://doi.org/10.1007/BF01172967

Gallwey, W. T. (1974). The inner game of tennis. New York, Estados Unidos: Random House.

Garcés de los Fayos, E. J. (1999). Un estudio de la influencia de variables de personalidad, sociodemográficas y deportivas en el sindrome de burnout. (Tesis doctoral). Universidad de Murcia, Murcia.

Gardner, F. L. y Moore, Z. (2004). A Mindfulness-acceptance-commitment-based approach to athletic performance enhancement: theoretical considerations. Behaviour Therapy, 35, 707723. https://doi.org/10.1016/S0005-7894(04)80016-9

Gardner, F. L. y Moore, Z. E. (2007). The psychology of enhancing human performance: The mindfulness-acceptance-commitment (MAC) approach. Nueva York, NY, Estados Unidos: Springer Publishing Company.

Goodman, F. R., Kashdan, T. B., Mallard, T. T. y Schumann, M. A. (2014). Brief Mindfulness and Yoga Intervention With an Entire NCAA Division I Athletic Team: An Initial Investigation. Psychology of Consciousness: Theory, Research, and Practice, 1, 339356. https://doi.org/10.1037/cns0000022 
Hanrahan, S. J., Grove, J. R. y Hattie, J. A. (1989). Development of a questionnaire measure of sport-related attributional style. International Journal of Sport Psychology, 20, 114-134.

Hardiness Institute (1985). Personal Views Survey. Arlington Heights, IL, Estados Unidos: Autor.

Hatzigeorgiadis, A. y Biddle, S. J. (2000). Assessing cognitive interference in sport: Development of the Thought Occurrence Questionnaire for Sport. Anxiety, Stress, and Coping, 13, 65-86. https://doi.org/10.1080/10615800008248334

Hayes, S. C. y Strosahl, K. D. (2004). Acceptance and commitment therapy, relational frame theory, and the third wave of behavioral and cognitive therapies. Behavior Therapy, 35, 639-665. https://doi.org/10.1016/S0005-7894(04)80013-3

Hayes, S. C., Strosahl, K. D. y Wilson, K. G. (1999). Acceptance and commitment therapy. Nueva York, NY, Estados Unidos: Guilford Press.

Henry, J. D. y Crawford, J. R. (2005). The shortform version of the Depression Anxiety Stress Scales (DASS-21): Construct validity and normative data in a large non-clinical sample. British Journal of Clinical Psychology, 44, 227-239. https://doi.org/10.1348/014466505X29657

Jackson, S. A. y Eklund, R. C. (2002). Assessing flow in physical activity: The Flow State Scale-2 and Dispositional Flow Scale-2. Journal of Sport and Exercise Psychology, 24, 133-150. https:ll doi.org/10.1123/jsep.24.2.133

Jackson, S. A. y Eklund, R. C. (2004). The flow scales manual. Morgantown, WV, Estados Unidos: Fitness Information Technology.

Jacobson, N. S., Christensen, A., Prince, S. E., Cordova, J. y Eldridge, K. (2000). Integrative Behavioral Couple Therapy. An acceptance-based, promising new treatment for couple discord. Journal of Consulting and Clinical Psychology, 68, 351-355. https://doi.org/10.1037/0022-006X.68.2.351

Jacobson, N. S., Martell, C. R. y Dimidjian, S. (2001). Behavioral activation treatment for depression: Returning to contextual roots. Clinical Psychology: Science and Practice, 8, 255-270. https://doi.org/10.1093/clipsy.8.3.255

John, S., Verma, S. K. y Khanna, G. L. (2011). The effect of mindfulness meditation on HPA-axis in pre-competition stress in sports performance of elite shooters. National Journal of Integrated Research and Medicine, 2(3), 15-21.

Kabat-Zinn, J. (1990). Full catastrophe living: using the wisdom of your body and mind to face stress, pain, and illness. New York, NY, Estados Unidos: Delta.

Kabat-Zinn, J. (2003). Mindfulness-based interventions in context: Past, present, and future. Clinical Psychology: Science and Practice, 10, 144-156. https://doi.org/10.1093/clipsy.bpg016

Kaufman, K. A. y Glass, C. R. (2006). Mindful Sport Performance Enhance ment: A treatment manual for archers and golfers. (Unpublished manuscript) The Catholic University of America, Washington D.C.

Kohlenberg, R. J. y Tsai, M. (1991). Functional analytic psychotherapy: A guide for creating intense and curative therapeutic relationships. Nueva York, NY, Estados Unidos: Plenum. https://doi. org/10.1007/978-0-387-70855-3

Lamers, S. M., Westerhof, G. J., Bohlmeijer, E. T., Klooster, P. M. y Keyes, C. L. (2011). Evaluating the psychometric properties of the mental health continuum-short form. (MHC-SF). Journal of Clinical Psychology, 67(1), 99-110. https://doi.org/10.1002/ jclp.20741

Lau, M. A., Bishop, S., Segal, Z., Buis, T., Anderson, N., Carlson, L., ... Devins, G. (2006). The Toronto Mindfulness Scale: Development and validation. Journal of Clinical Psychology, 62, 14451467. https://doi.org/10.1002/jclp.20326

Linehan, M. (1993). Cognitive behavioural treatment for borderline personality disorder. Nueva York, NY, Estados Unidos, NY, Estados Unidos: Guilford Press.

MacDonald L. A. y Minahan, C. L. (2018). Mindfulness training attenuates the increase in salivary cortisol concentration associated with competition in highly trained wheelchairbasketball players. Journal of Sports Sciences, 36, 378-383.

MacDonald, L. A., Oprescuc, Fy Keanb, B. M. (2018). An evaluation of the e ects of Mindfulness training from the perspectives of wheelchair basketball players. Psychology of Sport \& Exercise. 37, 188-195. https://doi.org/10.1016/j.psychsport.2017.11.013

Manzo, L. G., Silva, J. M y Mink, R. (2001). The Carolina Sport Confidence Inventory. Journal of Applied Sport Psychology, 13, 260274. https://doi.org/10.1080/104132001753144400

Mañas, I., Del Águila, J., Franco, C., Gil, M. D. y Gil, C. (2014). Mindfulness y rendimiento deportivo. Psychology, Society, \& Education, 6(1), 41-53. https://doi.org/10.25115/psye.v6i7.507

Marcus, B., Selby, V., Niaura, R. y Rossi. J. (1992). Self-efficacy and the stages of exercise behavior change. Research Quarterly for Exercise and Sport, 63(1), 60-66. https://doi.org/10.1080/02701 367.1992.10607557

Martin, G. L. y Toogood, A. (1997). Cognitive and behavioral components of a seasonal psychological skills training program for competitive figure skaters. Cognitive and Behavioral Practice, 4, 383-404. https://doi.org/10.1016/S1077-7229(97)80008-9

Palmi, J. y Solé, S. (2016). Intervenciones basadas en mindfulness (atención plena) en Psicología del Deporte. Revista de Psicología del Deporte, 25(1), 147-155.

Perestelo, L. (2013). Standard son how to develop and report systematic reviews in Psychology and Health. International Journal of Clinical and Health Psychology, 13, 49-57. https://doi. org/10.1016/S1697-2600(13)70007-3

Pérez-Álvarez, M. (2012). Third-generation Therapies: achievements and challenges. International Journal of clinical and Health Psychology, 12(2), 291-310.

Pineau, T., Glass, C. y Kaufman, K., (2014). Mindfulness in Sport Performance. Handbook of mindfulness. Oxford (UK). Wiley-Blackwell.

Rossi, J. (1992). Self-Efficacy and the Stages of Exercise Behavior Change. Research Quarterly for Exercise and Sport, 63, 60-66. https://doi.org/10.1080/02701367.1992.10607557

Ryff, C. y Keyes, C. (1995). The structure of psychological well-being revisited. Journal of Personality and Social Psychology, 69, 719-727. https://doi.org/10.1037/0022-3514.69.4.719

Scott -Hamilton, J., Schutte N. S. y Brown, R. (2016). Effects of a Mindfulness Intervention on SportsAnxiety, Pessimism, and Flow in Competitive Cyclist. Applied Psychology: Health and WeII-Being, 8(1), 85-103. https://doi.org/10.1171/aphw.12063

Segal, Z. V., Williams, J. M. y Teasdale, J. D. (2002). Mindfulness-based cognitive therapy for depression. New York: Guilford Press.

Shapiro, S. L. y Schwartz, G. E. (1999). Intentional systemic mindfulness: an integrative model for self-regulation and health. Advances in Mind-Body Medicine, 15, 128-134. 
Slagter, H. A., Lutz, A., Greischar, L., Francis, A., Nieuwenhuis, S., Davis, J. y Davidson, R. (2007). Mental training affects distribution of limited brain resources. PLoS Biology, 5 (138). https:// doi.org/10.1371/journal.pbio.0050138

Smith, R. E., Smoll, F. L., Cumming, S. P. y Grossbard, J. (2006). Measurement of multidimensional sport performance anxiety in children and adults: The Sports Anxiety Scale-2. Journal of Sport and Exercise Psychology, 28, 479-501. https://doi.org/10.1123/ jsep.28.4.479

Smith, R. E., Smoll, F. L y Schutz, R. W. (1990). Measurement and correlates of sportspeci!c cognitive and somatic trait anxiety: The Sport Anxiety Scale. Anxiety Research, 2, 263-280. https:/l doi.org/10.1080/08917779008248733

Snyder, C. R., Harris, C., Holleran, S., Irving, L., Sigmon, S., Yoshinobu, L., ... Harney, P. (1991). The will and the ways: Development and validation of an individual-differences measure of hope. Journal of Personality and Social Psychology, 60, 570-585. https://doi.org/10.1037/0022-3514.60.4.570

Thakur, T.S. y Mahesh, C. (2016). Enhancement in Shooting ability of Basketball players through Meditation. Research Journal of Physical Education Sciences, 4(4), 1-4.

Thompson, R. W., Kaufman, K. A., De Petrillo, L. A., Glass, C. R. y Arnkoff, D. B. (2011). One Year Follow-Up of Mindful Sport Performance Enhancement (MSPE). With Archers, Golfers, and
Runners. Journal of Clinical Sport Psychology, 5, 99-116. https:// doi.org/10.1123/jesp. 5.2.99

Watson, D. C., Clark, L. A. y Tellegen, A. (1988). Development and validation of brief measures of positive and negative affect: The PANAS scales. Journal of Personality and Social Psychology, 54, 1063-1070. https://doi.org/10.1037/0022-3514.54.6.1063

Wilson, K. G. y Luciano, M. C. (2002). Terapia de Aceptación y Compromiso (ACT): Un tratamiento conductual orientado a los valores. Madrid, España: Pirámide.

Wilson, K. G., Sandoz, E. K., Kitchens, J. y Roberts, M. (2011). The Valued Living Questionnaire: Defining and measuring valued action within a behavioral framework. The Psychological Record, 60, 249-272. https://doi.org/10.1007/BF03395706

Wininger, S. R. y Gieske, D. (2010). Measure of Attentional Focus: Cognitive Interviews and a Field Study. Athletic Insight, 2(2), 125-146.

Zeidan, F., Johnson, S. K., Diamond, B. J. y Goolkasian, P. (2010). Mindfulness meditation improves cognition: Evidence of brief mental training. Consciousness and Cognition, 19, 597-605. https://doi.org/10.1016/j.concog.2010.03.014

Zhang, C. Q., Si, G., Duan, Y., Lyu, Y., Keatley, D. y Chan, D. (2015). The effects of mindfulness training on beginners' skill acquisition in dart throwing: A randomized controlledtrial. Psychology of Sport \& Exercise, 22. https://doi.org/10.1016/j.psychsport.2015.09.005 